\title{
ANALYSIS OF IMAGE STORAGE AND RETRIEVAL IN GRADED MEMORY
}

\author{
B Sudarshan ${ }^{1}$, R Manjunatha $^{2}$ \\ ${ }^{I}$ Research Scholar, Electronics and Communication Engg, Department, Jain University, Bangalore, India \\ ${ }^{2}$ Research Guide, Electronics and Communication Engg, Department, Jain University, Bangalore, India
}

\begin{abstract}
An approach to storing and retrieving static images using multilayer Hopfield neural network is analyzed. Here, the Hopfield network is used as a memory, which stores images in predefined resolution. During the image retrieval, down sampled version of the stored image is provided as the query mage, The memory initially gives out a coarse image. The finer details of the image are synthesized later by using this coarse output image. This coarse output image is fed as the input to the memory again. The output this time will be better than the output that was got initially. The output of the memory becomes better and better as the time progresses. We call this memory a graded memory. Here the work proposes various models of the graded memory using multilayer Hopfield neural network, analyses the effectiveness of this memory with parameters like MSE, RMSE and PSNR.
\end{abstract}

Keywords: Hopfield network, graded memory, image storage, image retrieval.

\section{INTRODUCTION}

There are many ways in which picture images can be stored in memory and retrieved. Simple way is to store the image in normal memory without compressing the image. But, generally, conventional way of storing static images is by using some compression techniques like JPEG, Wavelets etc. This requires entire image to be compressed using any of the above mentioned technique then, coding this compressed data using suitable coding techniques.

The paper analyzes a different approach to static image storage and retrieval using a multilayer Hopfield neural network so that this can be used as a graded memory ${ }^{1}$. Here, a single multilayer Hopfield neural network acts like a memory by storing multiple images. The stored images can be retrieved by providing the corresponding down sampled image as the input to the neural network. The image thus retrieved may be lossy, i.e., the retrieved image may not exactly match pixel to pixel. The lossy image is sufficient in many situations to arrive at a conclusion. The quality of the image recall is analyzed using parameters like Mean Squared Error (MSE), Root Mean Squared Error (RMSE) and the Peak Signal to Noise ratio (PSNR).

Few authors have proposed storing gray scale images[3][4] using Hopfield neural network. The method presented by Giovanni Costantini[4] et al. decomposes the gray scale image into L binary patterns. Each pattern represents one bit in a digital coding of the gray levels. The image is stored independently using a conventional neural binary network with $\mathbf{n}$ neurons, where $\mathbf{n}$ is the number of pixels. There are $\mathrm{L}$ uncoupled neural networks with $\mathbf{n}^{2}$ connections in each level. The main advantage is that $\mathrm{L}$ uncoupled neural networks can be implemented in parallel saving considerable amount of time during both training as well as during recall. In this method, if a binary pattern cannot be stored in one sub-network, then the whole image cannot be stored. C. Oh[5] et al. proposed design of associative memory, wherein a large scale image is decomposed into many sub-images \& stored in independent neural networks. Similar problem arise in this method also. Overlapping between sub-images is done to overcome these effects. Igor Aizenberg[2] et al. proposed multilayer neural network with multi valued to classify textures.

In the proposed work, the multilayer Hopfield network is used as memory and the algorithm presented in[3] is adapted. This algorithm is better than the one used in[4] which stores an image in the neural network having $\mathrm{L}$ uncoupled layers without any interaction among the layers.

The paper is organized as below. A brief of Hopfield neural network is discussed in Section 2. Section 3 elaborates the actual design of graded memory focusing on image storage and retrieval. Section 4 explains simulation setup and results. Section 5 provides conclusion and future scope of work.

\section{MULTILAYER HOPFIELD NEURAL NETWORK}

Multilayer 2-dimensional Hopfield neural network[6] has only one layer of neurons. Each neuron has an input, an output and also performs computation. All neurons in the layer have bidirectional interconnections. The state equation of the network is given by

$\frac{d U_{i j}}{d t}=-U_{i j}+\sum_{k l} W_{i j, k l} V_{k l}+I_{i j}$

where, $\mathrm{i}, \mathrm{k}=0,1,2 \ldots \mathrm{M}$ and $\mathrm{j}, \mathrm{l}=0,1,2 \ldots \mathrm{N}$ 
$\mathrm{MxN}$ is the number of neurons, $\mathrm{U}_{\mathrm{ij}}$ is the input to the neuron at $\mathrm{i}^{\text {th }}$ row and $\mathrm{j}^{\text {th }}$ column. $\mathrm{V}_{\mathrm{kl}}$ is the neuron output at $\mathrm{i}^{\text {th }}$ row and $j^{\text {th }}$ column. $W=\left[\mathrm{W}_{\mathrm{ij}, \mathrm{kl}}\right]$ is the weight matrix for connections. Input bias $I_{i j}$ is taken as zero for all $\mathrm{i}, \mathrm{j}$ here. The output $\mathrm{V}_{\mathrm{ij}}$ is found by applying piecewise-linear saturation function $\mathrm{f}(\mathrm{x})=0.5(|\mathrm{x}+1|-|\mathrm{x}-1|)$. In this work, to store a gray scale image, 3-dimensional (MxNxL) multilayer Hopfield neural network is considered, where $\mathbf{M}$ denotes number of rows, $\mathrm{N}$ columns and $\mathrm{L}$, the levels in the grid of 3-dimensional neurons, the current neuron is denoted by $\mathrm{C}(\mathrm{i}, \mathrm{j}, \mathrm{p})$ where $\mathrm{i}, \mathrm{j} \& \mathrm{p}$ denote row, column and level respectively. Each neuron is connected to other neurons in the neighborhood given by the following constraint as presented $\mathrm{in}^{3}$.
$N_{r, s}(i, j, p)=\{(k, 1, q):|k-q| \leq r \quad v|k-I \geq M-r| 1-,j|\leq r v| 1-j|\geq N-r| q-$, $\mathrm{p} \| \leq \mathrm{s}$ v $|\mathrm{q}-\mathrm{p}| \geq \mathrm{L}-\mathrm{s}\}$

Each neuron is connected to $(2 r+1)^{2}$ neurons in the nearest neighborhood defined by the above constraint in each of the layers $p-s, \ldots, p-1, p, p+1, \ldots, p+s$, and $(2 s+1)$ connections in other layers. Here $r \geq s$ is assumed. Each neuron will have $\mu$ number of connections given by $\mu=(2 s+1)(2 r+1)^{2}$

The above constraint defining the neighborhood makes the network to have wraparound connections, meaning neurons lying on a torus with wraparound connections. For the neural network without wrap around connections, i.e., nontorus neural network, the equation (2) reduces to

$$
N_{r, s}(i, j, p)=\{(k, 1, q):|k-q| \leq r,|1-j| \leq r, \mid q-p \| \leq s\}
$$

The state equation for this 3-dimensional Hopfield neural network having $\mathrm{L}$ number of 2-dimensional layers is given by

$$
\frac{d U_{i j p}}{d t}=-U_{i j p}+\sum_{C(k, l, q) \in N_{r, s}(i, j, p)} W_{i j p, k l q} V_{k l q}+I_{i j p}
$$

The image with $n$ pixels and $2^{L}$ gray levels is decomposed into $L$ binary patterns with $n$ pixels. Each pattern corresponds to a layer of the multilayer Hopfield neural network. Hence, the proposed architecture consists of $L$ layers, each with $n$ neurons; the total number of neurons is $n L$ and the total number of interconnections is $\mu n \mathrm{~L}$. The number of interconnections grows only linearly with the number of pixels and logarithmically with the number of gray levels. The image is decomposed into binary patterns using binary weighted code. These are then converted to bipolar patterns and these bipolar patterns are used to train the neural network which is discussed in the next section. It is observed that binary patterns coded in reflective gray coding technique did not give better results compared to when binary patterns coded using binary weighted code. Whereas, in[3], binary patterns are coded in reflective gray coding technique.

\section{DESIGN OF GRADED MEMORY}

\subsection{Image Storage}

To store the gray scale image in a memory, the images shown figure 1 are taken, since storing a $128 \times 128$ sized gray scale image requires large number of neurons and connections requiring huge computer resources. Due to computer resource issues related to virtual memory allotment for storing large number of connections, the grayscale image to be stored is partitioned into many sub-blocks of equal size. For example, 128x128 image can be partitioned into 16 sub-blocks of size $32 \times 32,64$ sub-blocks of $16 \times 16$ size or 256 sub-blocks of size $8 \times 8$. When an image of size $48 \times 48$ is partitioned into 9 sub-blocks of size $16 \times 16$ pixels each, the neural network for all these 9 sub-blocks of the image is trained simultaneously. Each of these subimages are stored in the neural network during training. The method used to design the multilayer Hopfield network is adapted from ${ }^{3}$ is as given below for reference.

The simulation setup includes simulation of training and testing of the multilayer Hopfield Neural network using MATLAB 7.10. The values of delta is set to 500, $\mathrm{r}$ and $\mathrm{s}$ values are set to 4 with learning rate taken as 1 .

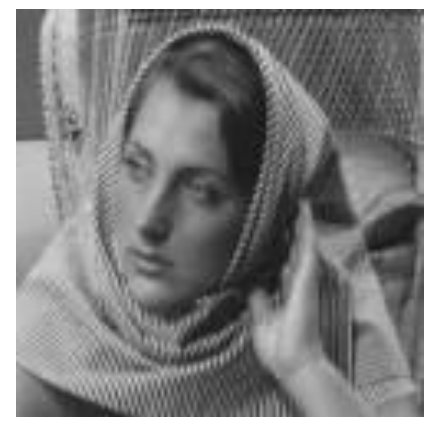

(a)

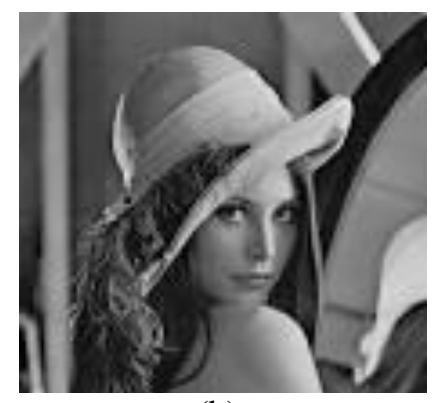

(b)

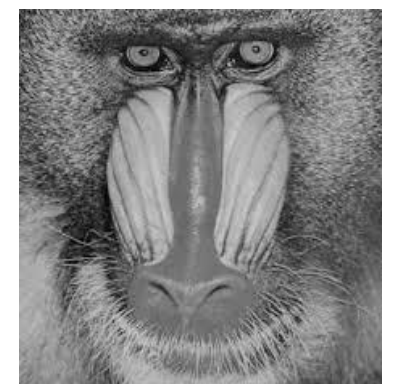

(c)

Fig 1 images used for training the neural network 
Let $\mathrm{y}_{\mathrm{ijp}}=\mathrm{f}\left(\mathrm{x}_{\mathrm{ijp}}\right)$

Assume $\mathrm{W}_{\mathrm{ijp}, \mathrm{klq}}=1$ for all $\mathrm{i}, \mathrm{j}, \mathrm{p}=\mathrm{k}, \mathrm{l}, \mathrm{q}$.

If there are Q images to be stored in the network, then each gray scale image coded in binary weighted code is first decomposed into L binary patterns, these patterns are then converted to bipolar patterns with 0 replaced by -1 and retaining 1 as it is. The $\mathrm{i}^{\text {th }}$ image $\mathbf{y i} \in\{-1,+1\}^{M \times N \times L}$.

So $\mathrm{y}_{1}, \mathrm{y}_{2} \ldots \mathrm{y}_{\mathrm{Q}}$ are the $\mathrm{Q}$ bipolar patterns corresponding to the $\mathrm{Q}$ images to be stored.

Connection weights $\mathrm{W}_{\mathrm{ijp}, \mathrm{klq}}$ satisfying the following set of constraints are found.

The constraints are as below.

$\sum_{C(k, l, q) \in N_{r, s}(i, j, p)} W_{i j p, k l q} V_{i j p}^{(m)} V_{k l q}^{(m)} \geq \delta>0$

Where $\mathrm{I}, \mathrm{k}=1,2, \ldots \mathrm{M}, \quad \mathrm{j}, \mathrm{l}=1,2, \ldots . \mathrm{N}, \quad \mathrm{p}, \mathrm{q}=1,2, . . \mathrm{L}$ and $\mathrm{m}=$ $\mathrm{I}, 2, . . \mathrm{Q}$ and $\delta$ is the stability factor for the stored images in the network.

Connection weights are computed using the following algorithm.

Initially, all the weights are set to 0 , i.e., $\quad \mathrm{W}_{\mathrm{ijp}, \mathrm{klq}}(0)=0$ for all $\mathrm{i}, \mathrm{j}, \mathrm{p} \neq \mathrm{k}, \mathrm{l}, \mathrm{q}$

For every iteration, i.e., $\mathrm{t}>0$

$\Delta_{i j p}^{(m)}(t)=\sum_{C(k, l, q) \in N_{r, s}(i, j, p)} W_{i j p, k l q} V_{i j p}^{(m)} V_{k l q}^{(m)}-\delta$

Then, $\mathrm{P}\left(\Delta_{i j p}(t)\right)=0$ for $\Delta_{i j p}(t) \geq 0$ and $\mathrm{P}\left(\Delta_{i j p}(t)\right)=1$ for $\Delta_{i j p}(t)<0$ are calculated.

The connection weights are updated with the following equation.

$$
\begin{aligned}
& W_{i j p, k l q}(t+1) \\
& =W_{i j p, k l q}(t) \\
& +\eta \sum_{C(k, l, q) \in N_{r, s}(i, j, p)} V_{i j p}^{(m)} V_{k l q}^{(m)} \mathrm{P}\left(\Delta_{i j p}(t)\right)
\end{aligned}
$$

Where, $\eta$ is the learning rate greater than zero. Here the computed $\mathrm{W}$ matrix is not symmetric.

\subsection{Image Retrieval}

Once the network is trained to store all the sub-blocks of the entire image meeting the constraints specified in the algorithm, the stored images are retrieved by providing the down sampled version of the partitioned input image. During the retrieval of the complete image, the trained network is fed with down sampled version (test image) of the partitioned images one after the other. For each down sampled version of the input partition image that is fed to the network, the recalled image is initially a coarse output image, this coarse output is fed as the input to the network in the $2^{\text {nd }}$ pass and it is observed that the output obtained in the $2^{\text {nd }}$ pass is better than that obtained in the first pass. In the third pass, the output image obtained in $2^{\text {nd }}$ pass is fed as the input image and the third pass output is noted. This is repeated till the output image of some $\mathrm{a}^{\text {th }}$ pass is satisfactory. This is repeated for each partitioned image. After noting all the recalled images, these are combined to get the total image. The recalled combined image is compared against the stored image and the performance of the network is measured using the parameters MSE, RMSE and PSNR. The results are presented in section 4 .

\section{SIMULATION AND RESULTS}

Matlab code is written to simulate multilayer Hopfield neural network using the algorithm presented in section 3.1 and 3.2. The three images shown in figure 1 are used to test the performance of the graded memory. The graded memory stores 9 images of size 16x16 during the training and during the retrieval, don sampled version of these images are given as test images and the results are noted. Table 1 shows image retrieval when the image shown in fig. 1 (a) of size $48 \times 48$ is partitioned into 9 images and stored in the graded memory. Similarly, the tables 3 and 4 show the image retrieval results obtained when the images shown in fig. 1(b) and (c) are partitioned and stored .

Table 2 shows the consolidated image quality in terms of PSNR in $\mathrm{dB}$ when the multilayer Hopfield neural network is connected in fully connected, torus and non torus fashion Figures 2-4 show the graph showing the image retrieval quality in terms of PSNR in $\mathrm{dB}$ for images shown in fig 1

\begin{tabular}{|c|c|c|c|}
\hline $\begin{array}{l}\text { Training } \\
\text { image }\end{array}$ & & Test Image & \\
\hline & $\begin{array}{l}\text { Fully } \\
\text { Connected }\end{array}$ & $\begin{array}{l}\text { Torus } \\
\text { connection }\end{array}$ & $\begin{array}{l}\text { Non Torus } \\
\text { connection }\end{array}$ \\
\hline $\begin{array}{l}\text { Output } \\
\text { for tes } \\
\text { image }\end{array}$ & & & $-5,20$ \\
\hline $1^{\text {st }}$ pass & & & \\
\hline $2^{\text {nd }}$ pass & & & \\
\hline 3rd pass & & & \\
\hline $4^{\text {th }}$ pass & & & \\
\hline
\end{tabular}

Table 1 Image retrieval when network stores 9 images in different configuration of the network 


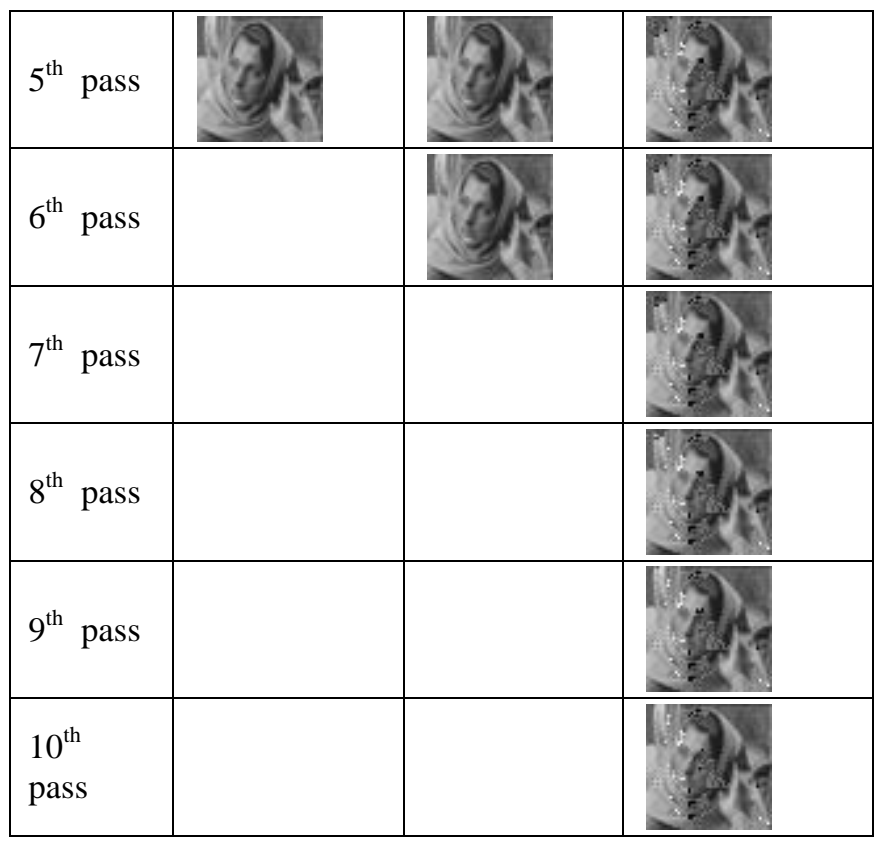

Table 2 PSNR(dB) values obtained when images are retrieved from graded memory, each row indicates the number of the iteration

\begin{tabular}{|l|l|l|l|l|l|l|l|l|}
\hline \multicolumn{1}{|l|}{ Barbara } & \multicolumn{1}{l|}{ Lena } & \multicolumn{3}{l|}{ Baboon } \\
\hline FC & T & NT & FC & T & NT & FC & T & NT \\
\hline 18.6 & 17.0 & 15.2 & 14. & 13.8 & 12.2 & 29.6 & 23.8 & 18.4 \\
\hline 22.9 & 22.0 & 17.4 & 17. & 15.7 & 12.6 & 100 & 38.1 & 23.2 \\
\hline 24.6 & 25.0 & 19.8 & 19. & 18.2 & 13.8 & & 100 & 24.4 \\
\hline 26.2 & 26.4 & 19.9 & 33. & 20.0 & 14.9 & & & 26.0 \\
\hline 45.6 & 40.6 & 20.4 & 100 & 21.4 & 15.8 & & & 26.0 \\
\hline 100 & 46.3 & 20.8 & & 25.1 & 17.5 & & & 26.1 \\
\hline & 46.3 & 20.8 & & 100 & 20.3 & & & 26.5 \\
\hline & 46.5 & 20.9 & & & 22.0 & & & 26.8 \\
\hline & & 21.3 & & & 22.1 & & & 27.0 \\
\hline & & 22.0 & & & 22.1 & & & 27.2 \\
\hline & & 22.3 & & & & & & 27.2 \\
\hline
\end{tabular}

where

FC- Fully Connected network

$\mathrm{T}$ - Torus network NT - Non Torus network

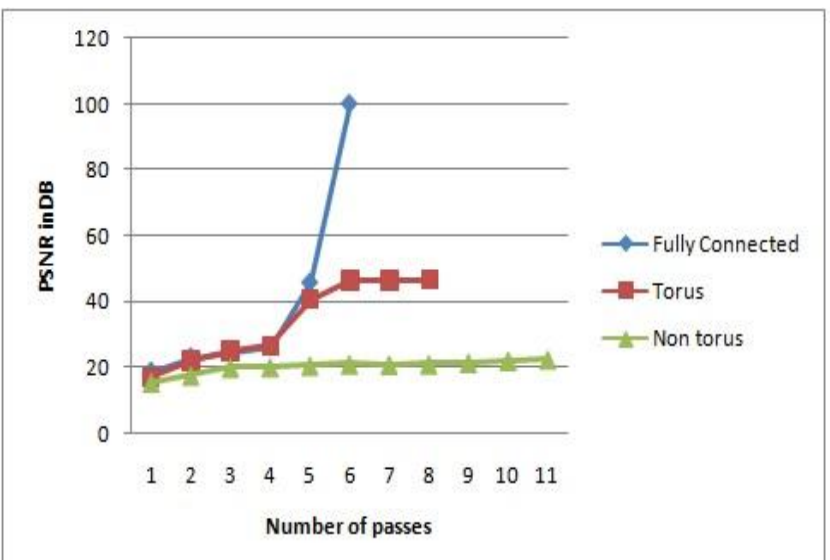

Fig 2 Graph showing PSNR variation with respect to type of network used for image shown in fig. 1. (a)
Table 3 Image retrieval when network stores 9 images in different configuration of the network

\begin{tabular}{|c|c|c|c|}
\hline \multirow[t]{2}{*}{$\begin{array}{l}\text { Training } \\
\text { image }\end{array}$} & loy & Test Image & \\
\hline & $\begin{array}{l}\text { Fully } \\
\text { Connected }\end{array}$ & $\begin{array}{l}\text { Torus } \\
\text { connection }\end{array}$ & $\begin{array}{l}\text { Non Torus } \\
\text { connection }\end{array}$ \\
\hline $\begin{array}{l}\text { Output } \\
\text { for test } \\
\text { image }\end{array}$ & (3) & & 8 \\
\hline $1^{\text {st }}$ pass & & & \\
\hline $2^{\text {nd }}$ pass & & & \\
\hline 3rd pass & & & \\
\hline $4^{\text {th }}$ pass & & & \\
\hline $5^{\text {th }}$ pass & & & \\
\hline $6^{\text {th }}$ pass & & & \\
\hline $7^{\text {th }}$ pass & & & \\
\hline $8^{\text {th }}$ pass & & & \\
\hline
\end{tabular}

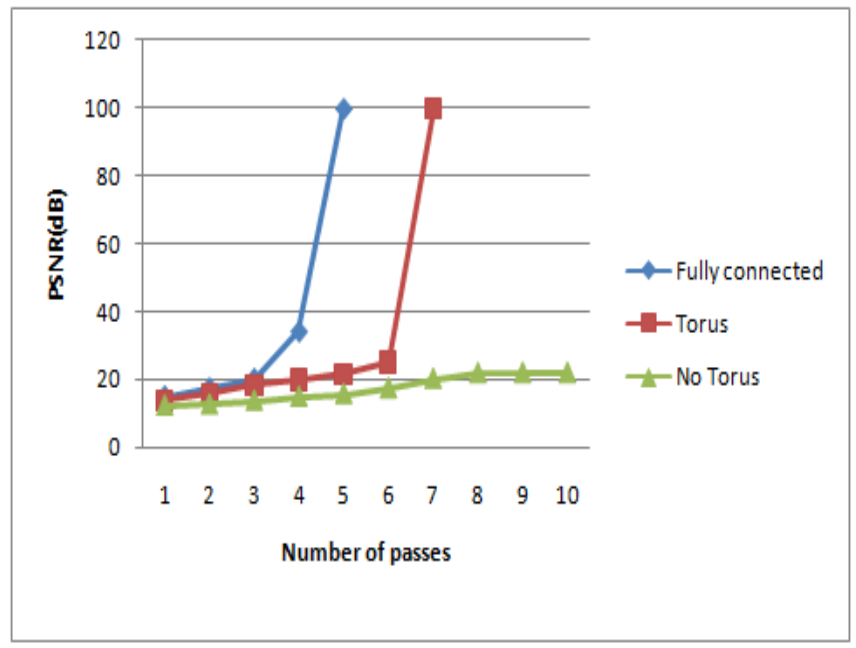

Fig 3 Graph showing PSNR variation with respect to type of network used for image shown in fig. 1.(b) 
Table 4 Image retrieval when network stores 9 images in different configuration of the network

\begin{tabular}{|l|l|l|l|}
\hline $\begin{array}{l}\text { Training } \\
\text { image }\end{array}$ & Test Image & Fully \\
Connected & $\begin{array}{l}\text { Torus } \\
\text { connection }\end{array}$ & $\begin{array}{l}\text { Non connection } \\
\text { corus }\end{array}$ \\
\hline $\begin{array}{l}\text { Output for } \\
\text { test image }\end{array}$ & & & \\
\hline $1^{\text {st }}$ pass & & & \\
\hline $2^{\text {nd }}$ pass & & & \\
\hline $9^{\text {th }}$ pass & & & \\
\hline $8^{\text {th }}$ pass & & & \\
\hline $6^{\text {th }}$ pass & & & \\
\hline $4^{\text {th }}$ pass & & & \\
\hline & & & \\
\hline
\end{tabular}

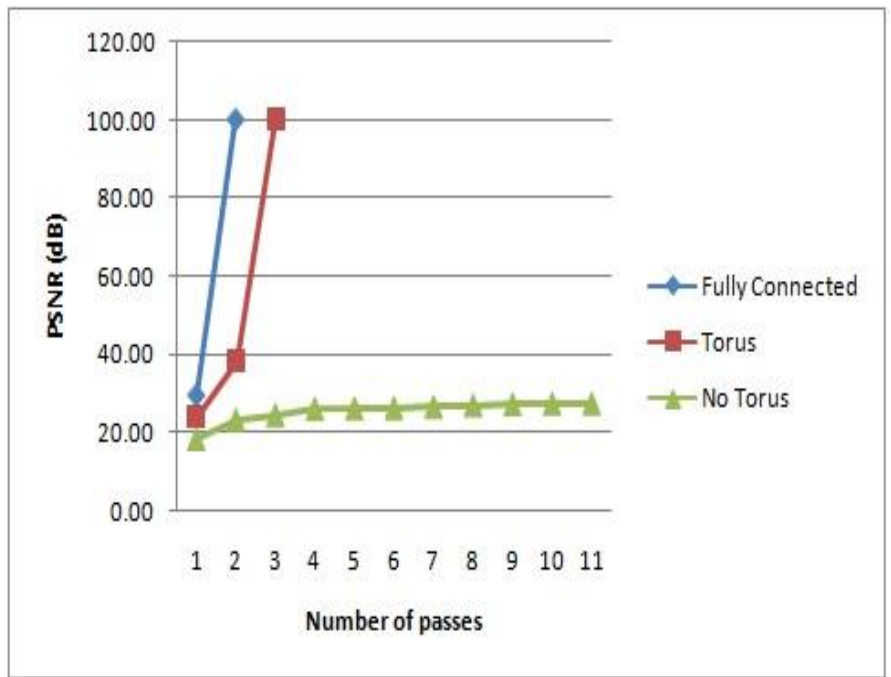

Fig 4 Graph showing PSNR variation with respect to type of network used for image shown in fig. 1.(c)

\section{CONCLUSION AND FUTURE WORK}

In this work, the multilayer Hopfield neural network has been used in three configurations i) Fully connected ii) Torus connection and iii) non torus connection. From the simulation results it is observed that multilayer Hopfield neural network can be configured to work as a graded memory in all these three configurations especially in fully connected fashion. It is clear from the results that the fully connected neural network generates the image that exactly match the original image in few passes whereas torus connected network is able to generate image that is enough to arrive at conclusion in few passes though it is not able to generate the original image exactly. The non torus connected network is able to generate the image which of poor quality compared to that obtained in other to cases. We can conclude that Fully connected network is best among the three network configurations used. The only limitation is that the network requires large number of neurons and connection weights.

Future work can focus on how to improve the quality of image when the network is connected in non torus fashion.

Also, the graded memory currently using the above algorithm is able to store and retrieve up to Nine images successfully, need to look at how to improve this number further.

\section{REFERENCES}

[1] B. Sudarshan, R Manjunatha, Image Storage and Retrieval in Graded Memory, International Journal of Advances in Engineering \& Technology (IJAET), Volume 8 Issue 1, Feb. 2015, pp. 2123-2128.

[2] Igor Aizenberg, Senior Member, IEEE, Jacob Jackson, and Shane Alexander, Classification of Blurred Textures using Multilayer Neural Network Based on Multi-Valued Neurons, Proc. of International Joint Conference on Neural Networks, San Jose, California, USA, July 31 - August 5, 2011. 
[3] Govanni Costantini, Design of Associative memory for Gray-Scale images by multilayer Hopfield Neural networks, Proc. Of the $10^{\text {th }}$ ESEAS international conference on CIRCUITS, Vouliagmeni, Athens, Greece, July 10-12, 2006, pp. 376-379.

[4] Giovanni Costantini, Daniele Casali and Renzo Perfetti, Neural associative memory storing grayscale images, IEEE Trans. Neural Networks, vol14, No. 3, May 2003, pp703-707.

[5] C. Oh, S H Zak, Associative memory design using overlapping decompositions and generalized brainstate-in a -box neural networks, Intl. Journal for Neural System. Vol. 13, no. 3, 2003, pp. 139-153.

[6] J.J. Hopfield, Neural networks and physical systems with emergent collective computational abilities, Proc. Nat. Acad. Sci., vol. 79, 1982, pp. 2554-2558.

\section{BIOGRAPHIES}

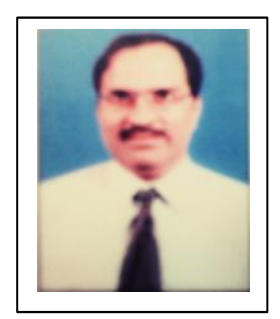

Mr. B Sudarshan is working as Assistant Professor at KS Institute of technology, Bangalore, India. $\mathrm{He}$ is currently pursuing $\mathrm{PhD}$ from Jain university, Bangalore India. He has more than 12 years of industrial experience in India and United states of America. His area of research includes image processing, artificial intelligence.

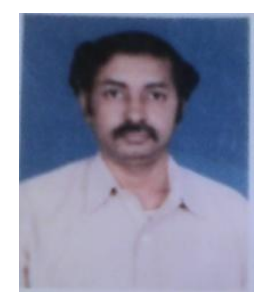

Dr. Manjunath $\mathrm{R}$ is well known scientist in India and abroad. He has got $\mathrm{PhD}$ from Bangalore university, India. He has 3 patents to his credit and published more than 100 papers in international journals. His research areas include DSP, artificial intelligence, image processing. 